\section{Cureus}

Received 01/07/2017

Review began 01/11/2017

Review ended 01/19/2017

Published 01/25/2017

(C) Copyright 2017

Amjad et al. This is an open access article distributed under the terms of the Creative Commons Attribution License CC-BY 3.0., which permits unrestricted use, distribution, and reproduction in any medium, provided the original author and source are credited.

\title{
Polycystic Liver Disease and Sarcoidosis: Unusual Coexisting Etiologies of Portal Hypertension
}

\author{
Waseem Amjad $^{1}$, Sophia Jagroop ${ }^{2}$, Rukma Parthvi ${ }^{2}$ \\ 1. Digestive Diseases, Mercy Medical Center, Baltimore, USA 2. Forest Hills Hospital, Northshore-Long \\ Island Jewish Health System
}

$\square$ Corresponding author: Waseem Amjad, waseemonline001@gmail.com Disclosures can be found in Additional Information at the end of the article

\section{Abstract}

Both polycystic liver disease (PLD) and sarcoidosis can involve liver. Most of the time, liver disease in both conditions is asymptomatic, but they can rarely cause portal hypertension. Our aim is to report a case of a 51-year-old female with a history of adult dominant polycystic kidney disease (ADPKD) and sarcoidosis who presented with multiple episodes of hematemesis. An endoscopy showed grade 3 esophageal varices. A computed tomography (CT) scan of the abdomen showed ascites with polycystic liver, nodular contour, and calcified granuloma. PLD can cause portal hypertension due to fibrosis or large cysts compressing on the portal vein. On the other hand, sarcoidosis causes portal hypertension by formation of arteriovenous (AV) shunts or fibrosis in areas of granulomas. Both conditions are diagnosed on imaging. There is no approved medical treatment for PLD; the only curative treatment is liver transplantation. Asymptomatic hepatic sarcoidosis does not need any treatment. The recommended treatment is corticosteroids for both isolated and systemic sarcoidosis. ADPKD and sarcoidosis can involve multiple organs. The presence of both conditions can accelerate the disease process and could be a therapeutic challenge. Early abdominal imaging during the course of both diseases can improve the outcome by decreasing the diagnostic window.

Categories: Gastroenterology

Keywords: sarcoidosis, polycystic liver disease, portal hypertesnion, cirrhosis

\section{Introduction}

Polycystic liver disease (PLD) is one of the rare conditions with a prevalence of $1 / 100,000$ to $1 / 1000,000$. Although typically asymptomatic, PLD can complicate the hemorrhage due to rupture of cysts, infection, and portal hypertension and, rarely, end stage liver disease [1]. There are very few cases in literature where polycystic liver disease presented with complication of portal hypertension. Sarcoidosis is a systemic granulomatous disease; it can involve the liver, but in most of the cases patients are asymptomatic. Cirrhosis and portal hypertension develop in less than one percent cases of sarcoidosis [2]. We present a case where adult dominant polycystic kidney disease (ADPKD) and sarcoidosis coexisted and presented as decompensated liver disease.

\section{Case Presentation}

A 51-year-old female presented to the emergency department after several episodes of hematemesis and severe epigastric pain. She had a past medical history of pulmonary sarcoidosis, ADPKD requiring bilateral nephrectomy and left kidney transplant, and is currently 
on immunosuppressive therapy. On admission, her vitals were stable. Her hemoglobin was 9.8 $\mathrm{g} / \mathrm{dl}$. Her total bilirubin was $0.2 \mathrm{mg} / \mathrm{dl}$, alkaline phosphates $74 \mathrm{U} / \mathrm{L}$, aspartate aminotransferase $24 \mathrm{U} / \mathrm{L}$, alanine aminotransferase $20 \mathrm{U} / \mathrm{L}$, blood urea nitrogen $49 \mathrm{mg} / \mathrm{dl}$, and creatinine 1.07 $\mathrm{mg} / \mathrm{dl}$. Electrolytes were normal. She underwent emergent esophagogastroduodenoscopy (EGD), which showed grade 3 esophageal varices and four of them were banded. Octreotide and pantoprazole drip were administered. A computed tomography (CT) scan of the abdomen showed ascites with polycystic liver, nodular contour, and several sites of calcifications (most likely calcified granulomas) (Figure 1). An abdominal ultrasound showed a heterogeneous liver with mild contour and patent portal circulation. A previous magnetic resonance imaging (MRI) of the pelvis, which was done two years ago, showed similar small cysts in the parenchyma; however, the calcifications and nodularity of the liver were new. Diagnostic paracentesis revealed serum to ascitic albumin gradient (SAAG) of 1.9 consistent with portal hypertension. The patient was started on non-selective beta blocker for stabilization of varices. Ceftriaxone was given for prophylaxis for spontaneous bacterial peritonitis. The patient's bleeding was controlled, and the hemoglobin level was stable during the hospital stay. She refused further workup including liver biopsy.

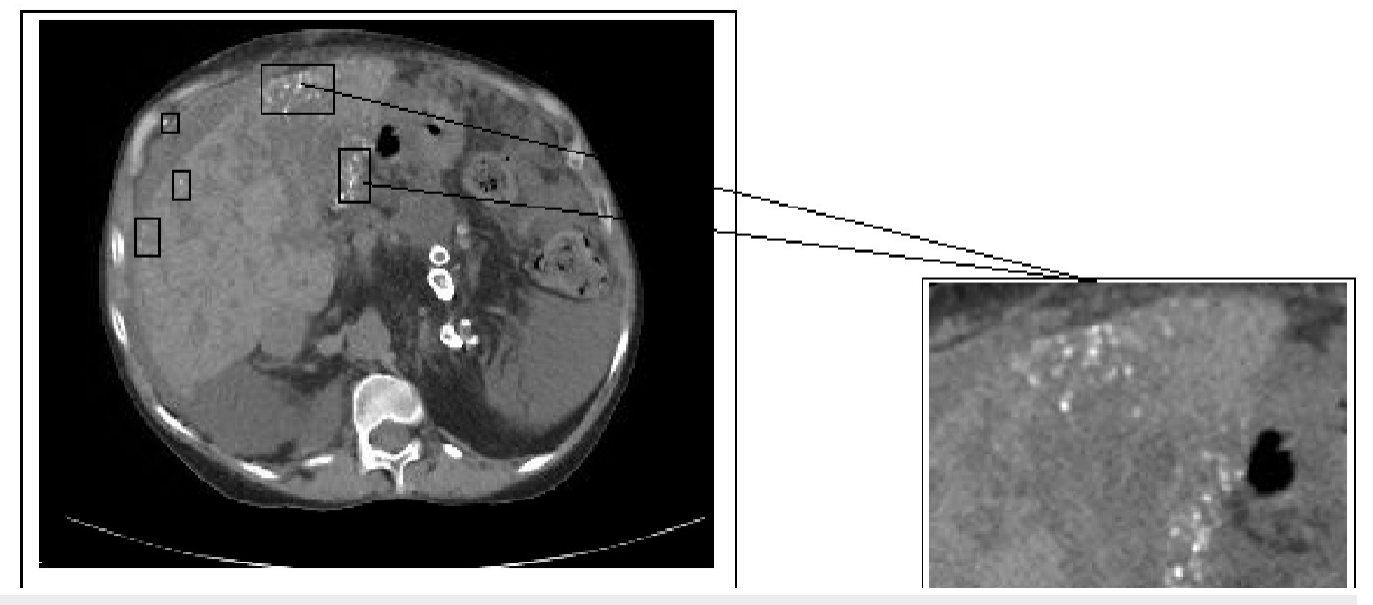

\section{FIGURE 1: Abdominal CT scan showing multiple cystic hypo- densities with calcification in liver parenchyma.}

\section{Discussion}

Polycystic liver disease occurs either in isolation or as part of ADPKD or uncommonly adult dominant polycystic liver disease (ADPLD). Polycystic liver disease involves mutation of polycystin 1, polycystin 2, hepatocystin, and Sec63, which leads to the failure of intralobular bile ducts to involute during embryonic development. The risk factors for hepatic cyst growth involve age, female gender, and size of renal cysts [3]. Hepatic cystic disease is usually asymptomatic and does not need intervention; rarely, extensive cystic disease may lead to liver failure. There are few cases in literature where polycystic liver disease complicated into portal hypertension either with fibrosis or with extensive cystic disease [4-5]. The pathogenesis includes two processes. First, polycystic disease decreases hepatic vein flow, and second cysts can compress the portal vein leading to portal hypertension [1].

Sarcoidosis is a systemic granulomatous disease that can commonly involve the liver. Hepatic sarcoidosis is usually asymptomatic, and rarely can it progress to cirrhosis, portal hypertension, cholestasis, and Budd-Chiari syndrome [6]. The pathophysiology of portal hypertension in sarcoidosis is unknown. Formation of AV shunts in areas of granulomas could be a cause; AV shunting increases the resistance which leads to portal hypertension. Another 
suggestion is that healing fibrosis in areas of granuloma increases the resistance, which can lead to portal hypertension with cirrhosis. Another theory suggests that ischemic changes caused by granulomatous phlebitis of portal and hepatic veins can lead to cirrhosis and portal hypertension [2].

Both conditions are diagnosed on imaging. Abdominal ultrasound and CT scan are the first line modalities. Liver biopsy supports diagnosis of sarcoidosis and rules out other conditions. Liver function abnormalities are common in symptomatic patients [3,6]. The presence of both sarcoidosis and polycystic liver disease increases the risk of development of decompensated liver disease or liver failure as compared to single disease. To our knowledge there is no case in literature where both conditions involved the liver simultaneously.

There is no approved medical treatment for PLD. Studies have shown somatostatin analogues and mammalian target of rapamycin (mTOR) inhibitors efficacy as compared to placebo. Radiological treatment includes aspiration followed by sclerotherapy for large cysts. Surgical treatment involves fenestration, segmental hepatic resection, and liver transplantation. Liver transplantation is the only curative treatment for PLD [3]. The Model of End Stage Liver Disease (MELD) score is commonly used for liver allocation. Usually liver functions are preserved in PLD, and the MELD score is not the best criteria to assess for liver transplantation. Some studies suggested the use of the MELD priority score to improve the outcome [7]. Asymptomatic hepatic sarcoidosis patients do not need any treatment. The recommended treatment is corticosteroid for both isolated and systemic sarcoidosis. Corticosteroids improve liver function tests but do not improve progression of disease. The survival in patients who have undergone a liver transplant with hepatic sarcoidosis is comparable to other liver diseases leading to transplant [8].

\section{Conclusions}

Polycystic liver disease and sarcoidosis are rare etiologies for portal hypertension.

The presence of both conditions can accelerate the disease process and could be a therapeutic challenge. An early abdominal imaging during the course of a disease can improve the outcome.

\section{Additional Information \\ Disclosures}

Human subjects: Consent was obtained by all participants in this study. Informed consent was obtained from the patient for this study. Conflicts of interest: In compliance with the ICMJE uniform disclosure form, all authors declare the following: Payment/services info: All authors have declared that no financial support was received from any organization for the submitted work. Financial relationships: All authors have declared that they have no financial relationships at present or within the previous three years with any organizations that might have an interest in the submitted work. Other relationships: All authors have declared that there are no other relationships or activities that could appear to have influenced the submitted work.

\section{References}

1. Cnossen WR, Drenth JP: Polycystic liver disease: an overview of pathogenesis, clinical manifestations and management. Orphanet J Rare Dis. 2014, 9:69. 10.1186/1750-1172-9-69

2. Blich M, Edoute Y: Clinical manifestations of sarcoid liver disease. J Gastroenterol Hepatol. 2004, 19:732-737. 10.1111/j.1440-1746.2003.03335.x

3. Chandok N: Polycystic liver disease: a clinical review . Ann Hepatol. 2012, 11:819-826.

4. Bandyopadhyay SK, Bandyopadhyay R, Dutta A: Adult polycystic liver disease presenting as 


\section{Cureus}

portal hypertension. J Indian Med Assoc. 2004, 102:384-385.

5. Rossi M, Spoletini G, Bussotti A, et al.: Combined liver-kidney transplantation in polycystic disease: case reports. Transplant Proc. 2008, 40:2075-2076.

10.1016/j.transproceed.2008.05.058

6. Tan CB, Rashid S, Rajan D, Gebre W, Mustacchia P: Hepatic sarcoidosis presenting as portal hypertension and liver cirrhosis: case report and review of the literature. Case Rep Gastroenterol. 2012, 6:183-189. 10.1159/000338355

7. Gringeri E, D’Amico FE, Bassi D, et al.: Liver transplantation for massive hepatomegaly due to polycystic liver disease: an extreme case. Transplant Proc. 2012, 44:2038-2040. 10.1016/j.transproceed.2012.06.041

8. Ebert EC, Kierson M, Hagspiel KD: Gastrointestinal and hepatic manifestations of sarcoidosis . Am J Gastroenterol. 2008, 103:3184-3192. 10.1111/j.1572-0241.2008.02202.x 